(C) 2017 IEEE. Personal use of this material is permitted. Permission from IEEE must be obtained for all other uses, in any current or future media, including reprinting/republishing this material for advertising or promotional purposes, creating new collective works, for resale or redistribution to servers or lists, or reuse of any copyrighted component of this work in other works. 


\title{
A Novel Method for Optimizing Distributed Generation in Distribution Networks Using the Game Theory
}

\author{
Arash Beiranvand ${ }^{1 *}$, Mohammad Abuhilaleh ${ }^{2}, \mathrm{Li} \mathrm{Li}^{2}$ \\ ${ }^{1}$ Department of Electrical Engineering, Shahid Beheshti University of Tehran, Tehran, Iran \\ ${ }^{2}$ Faculty of Engineering and IT, University of Technology Sydney, Australia \\ E-mail: arash.beiranvand65@gmail.com
}

\begin{abstract}
In this paper, a novel method is presented to optimize distributed generation (DG) in distribution networks. The suggested method shows how DGs should change their sizes and places, if it is necessary, to improve the voltage profile and total power loss of distribution networks. For this purpose, game theory is applied to model the optimization problem. At the first step, an appropriate game based on the Nash equilibrium is suggested. Using the specific features of game theory, the procedure of decision making in the operator centers of distribution grids is considered. Then, the optimization problem is solved by finding Nash equilibrium point. To solve the Nash equilibrium, a specific kind of genetic algorithm (GA) called Nash GA is applied.
\end{abstract}

\section{INTRODUCTION}

Distribution networks are one of the most important parts of today's power systems which distribute the received power from power transmission systems to local loads. Due to this important role, distribution networks have been attracting more and more attention in the research associated with power systems. Significantly, by applying new components in these grids such as DGs and energy storage systems, operators have had more new problems and consequently more research has been carried out in the literature about these specific problems. One of these important problems for operators is optimizing distribution networks to improve the quality of operation.

Optimizing distribution grids involves various aspects such as operation cost, voltage profile, reactive power, etc. For instance, in [1] a novel method is proposed to optimize reactive power in distribution networks which include photovoltaic units. In this paper, considering the power uncertainty from photovoltaic units, the integrated reactive power optimization of photovoltaic units and even capacitors is proposed, in order to keep the operation of distribution networks safe and economic. In [2], a new method to optimize maintenance time schedule in distribution networks is suggested. In this paper, using the NSGA-II algorithm with multi-period overall coding and considering mutually exclusive and simultaneous maintenance as the constraints, the optimization problem is solved. In [3], a new strategy is presented to enhance the reliability and quality of service in distribution systems. This paper suggests a mixed-integer nonlinear programming to find the optimal places of manual and automatic sectionalizing switches and protective equipment in distribution networks. The optimization problem in this paper has economic and technical aspects and the suggested algorithm considers both aspects to enhance the effectiveness of the proposed method. In [4], considering new features of today's distribution networks, a novel strategy is proposed to manage and optimize the operation of distribution networks. The presented method in this paper considers flexible demand devices such as electric vehicles or various household appliances, DGs and small energy storage systems as the specific features of such distribution networks. This paper reviews different aspects of the problem and suggests an approach for its solution.

To solve optimization problems, various optimization algorithms such as genetic algorithm (GA), particle swarm optimization (PSO), etc. could be applied. One of the specific method to solve optimization problems especially those with economic background is the game theory. Using the game theory in power systems studies has been considered in literature. In [5], using the game theory, a novel cooperative strategy is presented for micro-grids (MGs) of distribution networks. In this paper, to form coalitions, the suggested algorithm allows MGs to cooperate and self-organize into a partition composed of disjoint MG coalitions. In [6], the game theory is applied for finding the optimal contract between the owners of DGs. In this paper, at the first step using specific optimization algorithm called Pareto frontier differential evolution (PDE), the optimal places of DGs in the case study are selected, and then using the game theory, the optimal contract between the owners of DGs is found.

In this paper, a novel method is proposed to optimize DGs in distribution networks. The suggested method uses game theory to find optimal sizes and places for DGs. For this purpose, the paper suggests an adopted game based on the Nash equilibrium. Due to the specific features of game theory, the suggested method is able to consider all of important issues associated with distribution networks operation from different vantage points of operators. Consequently, considering the procedure of decision making in the operation centers of distribution networks becomes possible and this is one of the most important advantages of the suggested method in comparison with other methods in this field. To solve the Nash equilibrium a specific kind of GA called Nash GA is applied. Finding optimal solution by means of the Nash GA is faster than that from other optimization algorithms such as GA [7]. 
As a result, using the game theory makes the suggested method more flexible than other presented methods in this field.

The presenting procedure of this paper is as follows. At the first step, the Nash equilibrium is explained briefly. Then, based on the Nash equilibrium, an adopted game is suggested to optimize DGs in distribution systems. At the next step, a specific kind of GA called Nash GA is presented to solve the Nash equilibrium. Finally, using the case study, the presented method is evaluated.

\section{NASH EQUILIBRIUM}

Nash equilibrium is a specific optimization approach which was proposed by J. F. Nash [7]. Nash equilibrium is a non-cooperative game in the game theory which has two or more players. In this game, it is assumed that each player knows the equilibrium strategies of the other players, and in the Nash equilibrium point, each player gains nothing by changing only his/her own strategy [68]. It means that in the Nash equilibrium point no player can benefit by changing strategies while the other players keep theirs unchanged. Based on the mentioned points, the Nash equilibrium is expressed as follow:

Consider $(S, F)$ as a game with $\mathrm{M}$ players, where $\mathrm{S}$ is the strategy profiles and $F(x)$ is its payoff function calculated at $x \in S$. For $i \in\{1, \ldots, M\}, S_{i}$ is the strategy set of player (i):

$$
\begin{aligned}
& S=S_{1} \times S_{2} \times \ldots \times S_{M} \\
& F(x)=\left(f_{1}(x), f_{2}(x), \ldots, f_{M}(x)\right)
\end{aligned}
$$

$x_{i}$ is a strategy profile of player (i) and $x_{-i}$ is a strategy profile of all players except for player (i). Now, the strategy profile is a Nash equilibrium if:

$$
\forall i, x_{i} \in S_{i}: f_{i}\left(x_{i}^{*}, x_{-i}^{*}\right) \geq f_{i}\left(x_{i}, x_{-i}^{*}\right)
$$

In the case with two players, the Nash equilibrium could be presented as follows [7].

Assume $\mathrm{E}$ is the search space of the first player and $\mathrm{H}$ is the search space of the second player. A strategy $(\bar{x}, \bar{y}) \in E \times H$ is a Nash equilibrium if:

$$
\begin{aligned}
& f_{E}(\bar{x}, \bar{y})=\inf _{x \in E} f_{E}(x, \bar{y}) \\
& f_{H}(\bar{x}, \bar{y})=\inf _{y \in H} f_{H}(\bar{x}, y)
\end{aligned}
$$

\section{SugGeSted Method}

In this section, an adopted game is suggested to optimize DGs in distribution networks. The underlying optimization includes two factors: changing sizes and changing places of DGs.

\section{A. Suggested Game}

It is assumed that there are two vantage points in the operation center of distribution gird for optimizing DGs. One group believes the aim of optimization should be reducing total power loss of the gird, while another group believes the object of optimization should be improving the voltage profiles which means the voltages are close to 1 p.u. Player (1) represents the first group and Player (2) represents the second group. Now, they are going to find the optimal solution which satisfies both groups. It's clear this game is a Nash equilibrium game which should be solved. Both players present their own payoff functions based on their specific vantage points and try to maximize their payoffs.

The possible actions for both players are:

- Changing only the sizes of DGs within permissible ranges.

- Changing only the places of DGs among permitted buses

- Changing sizes and places of DGs

These potential actions could be presented as follow:

$$
\begin{aligned}
& S D_{i} \in\left(\alpha_{i}, \beta_{i}\right) \\
& S D=\left\{S D_{1}, S D_{2}, \ldots, S D_{N}\right\} \\
& X=\left\{x_{1}, x_{2}, \ldots, x_{M}\right\}
\end{aligned}
$$

Here $\mathrm{SD}_{\mathrm{i}}$ is the size of DG (i) and $\left(\alpha_{i}, \beta_{i}\right)$ is the set of permissible range for $\mathrm{DG}$ (i) and $\mathrm{SD}$ is the set of permissible sizes of DGs. Also, $\mathrm{X}$ is the set of the permitted buses.

Moreover, each player has his/her own cost prediction for changing the places of DGs. For a distribution network with (N) DGs, (K) buses and (L) lines, the payoff functions of two players are defined as follow:

$$
\begin{aligned}
& G_{1}=\left(\sum_{l=1}^{L} P_{\text {loss }, l}(S D, X)\right)_{p . u}+\left(\sum_{i=1}^{N} C_{1 i}\left(x_{i}\right)\right)_{p . u} \\
& G_{2}=\left(\sum_{k=1}^{K} \mid 1-V_{k}(S D, X)\right)_{p . u}+\left(\sum_{i=1}^{N} C_{2 i}\left(x_{i}\right)\right)_{p . u} \\
& x(i) \in X \\
& \text { payoffFunction }(1)=\frac{1}{G_{1}} \\
& \text { payoffFunction }(2)=\frac{1}{G_{2}}
\end{aligned}
$$

Here, $\mathrm{P}_{\text {loss, } 1}$ is the power loss of line $(l)$ and $\mathrm{V}_{\mathrm{k}}$ is the voltage of bus $(\mathrm{k}) ; \mathrm{C}_{1 \mathrm{i}}\left(\mathrm{x}_{\mathrm{i}}\right)\left(\mathrm{C}_{2 \mathrm{i}}\left(\mathrm{x}_{\mathrm{i}}\right)\right.$, resp.) is the Player (1)'s (Player(2)'s, resp.) cost prediction for transferring DG (i) from its current position to the suggested location i.e. $\mathrm{x}_{\mathrm{i}}$. The total cost of operating distribution gird could be considered as the base cost.

Both players apply the AC power flow (AC-PF) to calculate the effective factors in their payoff functions:

$$
\begin{aligned}
& S_{k}=P_{k}+j Q_{k}=V_{k}\left[\sum_{j=1}^{K} Y_{k j} V_{j}\right]^{*} \\
& V_{\min } \leq V_{k} \leq V_{\max },(k \in K) \\
& -f_{l}^{\max } \leq f_{l} \leq f_{l}^{\max },(l \in L) \\
& P_{D G, i}^{\min } \leq P_{D G, i} \leq P_{D G, i}^{\max } \\
& Q_{D G, i}^{\min } \leq Q_{D G, i} \leq Q_{D G, i}^{\max }
\end{aligned}
$$

where $f_{l}^{\max }$ is the maximum capacity of line $(l), P_{D G, i}$ 
and $Q_{D G, i}$ are the active and reactive power generated by DG (i) respectively. In those grids where the usual AC-PF is not converged due to the amount of $\mathrm{R} / \mathrm{X}$ ratio of lines, backward/forward sweep power flow is applied [9]. In this method, the total power loss of grid is calculated as follows:

$$
\begin{aligned}
& P_{\text {loss }}(k, k+1)=R_{k} \frac{P_{k}^{2}+Q_{k}^{2}}{V_{k}^{2}} \\
& Q_{\text {loss }}(k, k+1)=X_{k} \frac{P_{k}^{2}+Q_{k}^{2}}{V_{k}^{2}}
\end{aligned}
$$

where $\mathrm{P}_{\mathrm{k}}$ is real power flowing out of bus $(\mathrm{k}) ; \mathrm{Q}_{\mathrm{k}}$ is reactive power flowing out of bus $(\mathrm{k}) ; \mathrm{P}_{\text {loss }}(\mathrm{k}, \mathrm{k}+1)$ and $\mathrm{Q}_{\text {loss }}(\mathrm{k}, \mathrm{k}+1)$ are the active and reactive power losses between bus $(\mathrm{k})$ and bus $(\mathrm{k}+1)$ respectively.

The total power loss in feeder $(\mathrm{T})$ is calculated as follows:

$$
P_{T, \text { loss }}=\sum_{k=1}^{n} P_{\text {loss }}(k, k+1)
$$

where $\mathrm{n}$ is the number of buses in feeder (T).

The bus voltages are calculated in the forward sweep. Using the following equation in the forward sweep, the amount of magnitudes and phases are calculated:

$$
I_{k}=\frac{V_{k} \angle \delta_{k}-V_{k+1} \angle \delta_{k+1}}{r_{k}+j x_{k}}
$$

where $Z_{k}=r_{k}+j x_{k}$ is the impedance between bus $(\mathrm{k})$ and bus $(\mathrm{k}+1)$.

It's clear that the variables of the suggested game is SD and $\mathrm{X}$ :

$$
\begin{aligned}
& g_{1}=\frac{1}{G_{1}(S D, X)} \\
& g_{2}=\frac{1}{G_{2}(S D, X)} \\
& \text { payoffFunction }(1)=g_{1}(S D, X) \\
& \text { payoffFunction }(2)=g_{2}(S D, X)
\end{aligned}
$$

\section{B. Nash GA}

Finding the optimal solution for Nash equilibrium is difficult. Due to this fact, various methods have been suggested to solve this kind of game in the game theory. In [7], a new method applying the GA is proposed to solve Nash equilibrium. The procedure of the suggested method in this paper is as follows.

Consider $\mathrm{s}=\mathrm{AB}$ as the string representing the potential solution of an optimization problem with two objective functions, where A corresponds to the first criterion and $\mathrm{B}$ the second one. The optimization task of A represents Player (1) and the optimization task of B represents Player (2). Based on Nash theory, Player (1) optimizes s by modifying A, while B is fixed by Player (2). Similarly, Player (2) optimizes s by modifying B while A is fixed by Player (1).
Now, consider $A_{k-1}$ as the optimal value found by Player (1) at generation $\mathrm{k}-1$, and $\mathrm{B}_{\mathrm{k}-1}$ as the optimal value found by Player (2) at generation k-1. At generation $k$, Player (1) optimizes $A_{k}$ while using $B_{k-1}$ to evaluate $s$. Simultaneously, Player (2) optimizes $B_{k}$ while using $A_{k-1}$. After these steps, Player (1) sends the best value $A_{k}$ to Player (2) who will apply it at generation $k+1$. Similarly, Player (2) sends the best value $B_{k}$ to Player (1) who will apply it at generation $\mathrm{k}+1$. This procedure continues till both player wouldn't be able to continue improving their found solutions.

The Nash GA is applied to solve the suggested game in this paper. To this aim, the s is set as follow:

$$
s=\text { payoffFunction }(1) \times \text { payoffFunction }(2)=\left(\mathrm{b}_{1}\right)(S D, X) \times g
$$

\section{CASE STUdy}

The IEEE 33 buses standard network is applied as the case study of this paper. It is assumed that two DGs have been added to the grid at bus (13) and bus (23). The sizes of DG (1) and DG (2) are $0.2 \mathrm{MW}$ and $0.9 \mathrm{MW}$ respectively with the same P.F. $=0.6$. The grid is connected to the main power grid through bus (1).

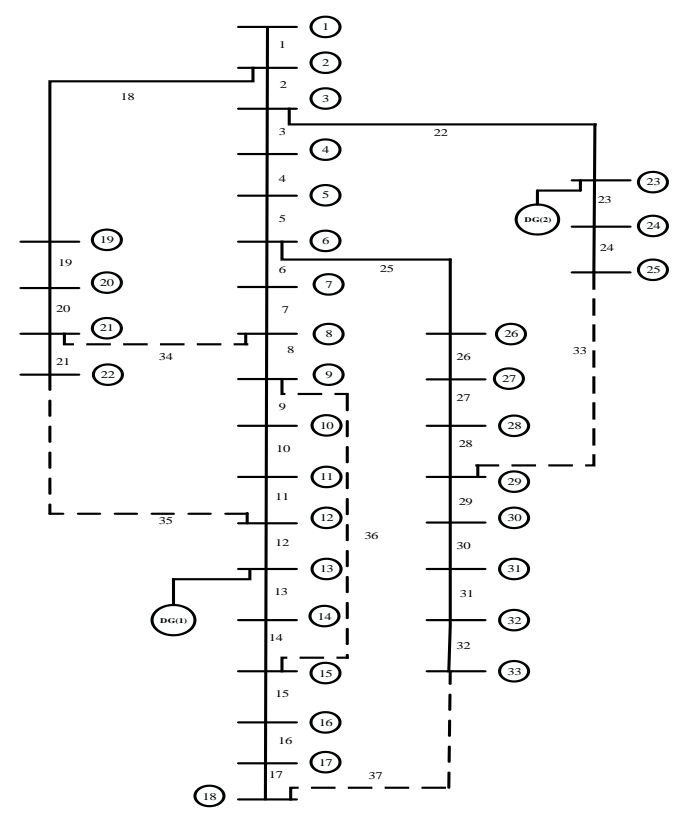

Fig. 1. The case study

More details about the case study could be found in the appendix and [10].

\section{Simulation}

\section{A. Simulation Results}

The predicted costs for changing the places of the DGs by each player can be found in Table I and Table II. It is assumed that buses 7, 20, 28, 30 are candidate buses in addition to the current corresponding buses of the DGs for the potential positions of the DGs. 
TABLE I

Cost prediction of Player 1 (p.u)

\begin{tabular}{|l|c|c|c|c|c|c|}
\hline Bus & & & & & & \\
\hline DG (1) & 7 & 13 & 23 & 20 & 28 & 30 \\
\hline DG (2) & 0.33 & 0 & 0.29 & 0.32 & 0.38 & 0.31 \\
\hline
\end{tabular}

TABLE II

Cost prediction of Player 2 (p.u)

\begin{tabular}{|c|c|c|c|c|c|c|}
\hline Bus & & & & & & \\
DG & 7 & 13 & 23 & 20 & 28 & 30 \\
\hline DG (1) & 0.09 & 0 & 0.11 & 0.13 & 0.25 & 0.23 \\
\hline DG (2) & 0.11 & 0.12 & 0 & 0.20 & 0.1 & 0.07 \\
\hline
\end{tabular}

Maximum possible size for both DGs is $2 \mathrm{MW}$. Also, the permissible range for bus voltages is $(0.94,1.06)_{p . u}$.

The solution of the suggested game obtained from solving the Nash GA can be found in Table III.

TABLE III

The optimal solution for the suggested game

\begin{tabular}{|c|c|c|}
\hline $\begin{array}{l}\text { solution } \\
\text { DGs }\end{array}$ & Size (MW) & Place \\
\hline DG (1) & 0.48 & 13 \\
\hline DG (2) & 0.51 & 30 \\
\hline
\end{tabular}

Fig. 2 shows the bus voltages before and after optimizing the grid by the suggested game. Also, Fig. 3 shows a comparison between the total power loss of the grid before and after optimizing the gird by the suggested game.

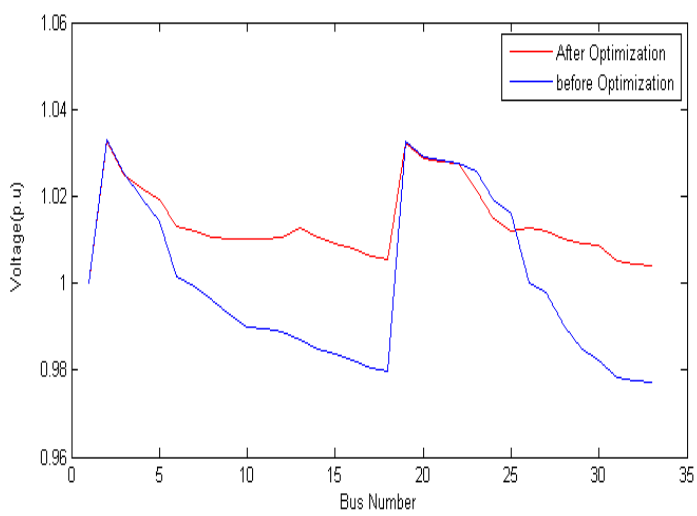

Fig. 2. Buses voltages before and after optimization by the suggested game

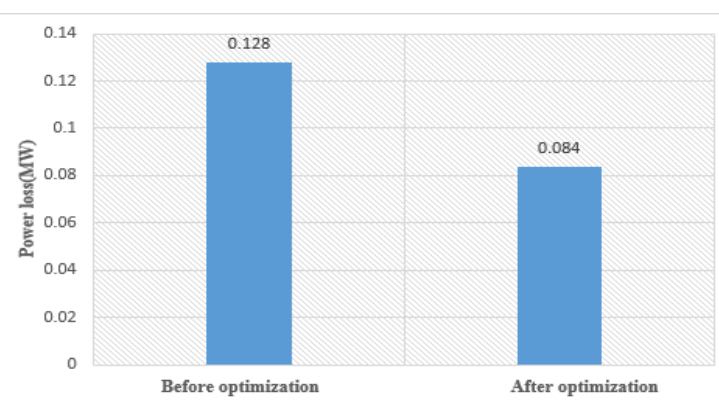

Fig. 3. Comparison between the total power loss of the grid before and after optimization

Fig. 2 shows the voltages at 22 buses are improved after optimizing the grid by the suggested game. Also, Fig. 3 shows the total power loss of the grid is reduced by
0.044 MW after optimization. The obtained results show the suggested method has succeeded in both objectives of the optimization problem.

\section{B. Discussion}

Probing the optimal solution obtained from solving the suggested game shows it is reasonable. With regards to the decision about DG (1), Player (1) has the high cost prediction for changing its place, and thus preferring not to change its place. Moreover, due to the fact that the buses placed at the end of feeders have worse voltage profiles in comparison with other buses, placing DGs in such zones helps to improve the voltage profile of the grid. Also, placing DGs in such zones is beneficial for decreasing the total power loss of the grid as well. Based on these points, not changing the place of DG (1) and increasing its size are reasonable for both players. For DG (2), bus (30) is suggested to be located at the end of the feeder, and as a result, this suggestion has the mentioned benefits as well. Also, as could be observed in Table I and Table II, the predicted costs by both players for transferring this DG to bus (30) are low. Consequently, this suggestion seems reasonable for both players too.

\section{CONCLUSION}

In this paper, using the game theory, a new method is proposed to optimize distributed generations in distribution grids. For this purpose, an adopted game based on Nash equilibrium is suggested. Due to specific features of the game theory, considering the procedure of decision making is possible and this feature is one of the most significant advantages of the suggested method in comparison with other methods in this field. Moreover, the suggested method considers the important issues associated with the operation of distribution networks and applies the AC-PF to calculate essential factors. The obtained results show the efficacy of the suggested method.

\section{APPENDIX}

The line information of IEEE 33-bus standard grid is listed in Table IV.

Table IV

Lines data of IEEE 33-bus

\begin{tabular}{|l|l|l|l|l|l|l|l|}
\hline $\begin{array}{l}\text { From } \\
\text { bus }\end{array}$ & $\begin{array}{l}\text { To } \\
\text { bus }\end{array}$ & R(p.u.) & X(p.u.) & $\begin{array}{l}\text { From } \\
\text { bus }\end{array}$ & $\begin{array}{l}\text { To } \\
\text { bus }\end{array}$ & R(p.u.) & X(p.u.) \\
\hline 2 & 3 & 0.03076 & 0.01567 & 2 & 19 & 0.01023 & 0.00976 \\
\hline 3 & 4 & 0.02284 & 0.01163 & 19 & 20 & 0.09385 & 0.08457 \\
\hline 4 & 5 & 0.02387 & 0.01211 & 20 & 21 & 0.02555 & 0.02985 \\
\hline 5 & 6 & 0.0511 & 0.04411 & 21 & 22 & 0.04423 & 0.05848 \\
\hline 6 & 7 & 0.01168 & 0.03861 & 3 & 23 & 0.02815 & 0.01924 \\
\hline 7 & 8 & 0.04439 & 0.01467 & 23 & 24 & 0.05603 & 0.04424 \\
\hline 8 & 9 & 0.06426 & 0.04617 & 24 & 25 & 0.0559 & 0.04374 \\
\hline 9 & 10 & 0.06514 & 0.04617 & 6 & 26 & 0.01267 & 0.00645 \\
\hline 10 & 11 & 0.01227 & 0.00406 & 26 & 27 & 0.01773 & 0.00903 \\
\hline 11 & 12 & 0.02336 & 0.00772 & 27 & 28 & 0.06607 & 0.05826 \\
\hline 12 & 13 & 0.09159 & 0.07206 & 28 & 29 & 0.05018 & 0.04371 \\
\hline 13 & 14 & 0.03379 & 0.04448 & 29 & 30 & 0.03166 & 0.01613 \\
\hline 14 & 15 & 0.03687 & 0.03282 & 30 & 31 & 0.0608 & 0.06008 \\
\hline 15 & 16 & 0.04656 & 0.034 & 31 & 32 & 0.01937 & 0.02258 \\
\hline 16 & 17 & 0.08042 & 0.10738 & 32 & 33 & 0.02128 & 0.03319 \\
\hline 17 & 18 & 0.04567 & 0.03581 & 1 & 2 & 0.00575 & 0.00293 \\
\hline
\end{tabular}




\section{REFERENCES}

[1] H. Yu, G. Shan, L. Siyuan, Z. Xin, H. Haiteng, "Reactive power optimization of distribution network including photovoltaic power considering harmonic factors", Electricity Distribution (CICED), 2016 China International Conference on, 10-13 Aug. 2016

[2] S. Pei-feng, M. Rui, C. Xing-ying, Y. Kun, Z. Dong-xu, L. Xin, X. Hong-hua, "Research on maintenance schedule optimization of distribution network including distributed generation", Electricity Distribution (CICED), 2016 China International Conference on, 10-13 Aug. 2016

[3] A. Heidari, V. G. Agelidis, M. Kia, J. Pou, J. Aghaei, M. Shafie-khah, J. P. S. Catalao, "Reliability Optimization of Automated Distribution Networks with Probability Customer Interruption Cost Model in the Presence of DG Units", IEEE Transactions on Smart Grid, 14. Sep. 2016

[4] E. Loukarakis, C. Dent, "Distribution network optimization for real-time generation and flexible demand management", Power Systems Computation Conference (PSCC), 20-24 June 2016

[5] W. Saad, Z. Han, H. V. Poor, "Coalitional Game Theory for Cooperative Micro-Grid Distribution Networks", Communications Workshops (ICC), 2011 IEEE International Conference on, 5-9 June 2011

[6] M. H. Moradi, M. Abedini, S. M. Hosseinian, "A Combination of Evolutionary Algorithm and Game Theory for Optimal Location and Operation of DG from DG Owner Standpoints", IEEE Transactions on Smart Grid, Vol.7, Issue. 2, pp: 608 - 616, 06 May 2015

[7] M. Sefrioui, J. Perlaux, "Nash Genetic Algorithms: examples and applications", Evolutionary Computation, 2000. Proceedings of the 2000 Congress on, 16-19 July 2000

[8] I. A. Ismail, N. A. El-Ramly, M. M. El-Kafrawy, M. M. Nasef, "Game Theory Using Genetic Algorithms", Proceedings of the World Congress on Engineering 2007 Vol. 1, July 2 - 4, 2007, London, U.K.

[9] J. A. Michline Rupa, S. Ganesh, "Power Flow Analysis for Radial Distribution System Using Backward/Forward Sweep Method", International Journal of Electrical, Computer, Energetic, Electronic and Communication Engineering Vol.8, No.10, 2014

[10] M. Khederzadeh, A. Beiranvand, "Identification and Prevention of Cascading Failures in Autonomous Microgrid", IEEE SYSTEMS JOURNAL, Oct, 16. 2015, DOI: 10.1109/JSYST.2015.2482227 